\title{
Longitudinal Assessment of Cognitive Function by Clock Drawing in Older Adults
}

\author{
Annlia Paganini-Hilla, b Linda J. Clark ${ }^{a}$ \\ ${ }^{a}$ Department of Preventive Medicine, Keck School of Medicine, University of Southern \\ California, Los Angeles, Calif., and ${ }^{b}$ Department of Neurology, University of California, \\ Irvine, Calif., USA
}

\section{Key Words}

Clock drawing $\cdot$ Cognitive function · Cognitive impairment $\cdot$ Cohort study $\cdot$ Dementia $\cdot$

Elderly $\cdot$ Longitudinal assessment $\cdot$ Mortality $\cdot$ Population-based study

\begin{abstract}
Aims: To test the hypotheses that performance on a clock-drawing task (CDT) diminishes over time and that poor performance is associated with development of dementia and reduced survival. Methods: Participants in the population-based Leisure World Cohort Study were asked to complete a CDT in follow-up questionnaires mailed in 1992 and 1998. Dementia status was determined using in-person evaluations, follow-up questionnaires, hospital data, and death certificates. Results: Of the 4,842 cohort members (mean age 80 years) who completed the CDT in 1992, 1,521 also completed it in 1998. Although performance in the CDT diminished over time in only a proportion of the cohort, the mean total score and the percentage of subjects with correctly drawn clocks decreased with each successive 5 -year age group $(p=0.0001)$ and over time. Mean scores were lower in non-demented subjects later identified as demented compared with those who remained free of dementia. A low CDT score in 1992 was associated with a $28 \%$ increased risk of dementia and $13 \%$ reduced survival. Conclusion: A CDT may help predict cognitive decline and future disability in the elderly. Identification of high-risk individuals using a CDT may lead to earlier diagnosis and treatment and improved patient management.
\end{abstract}

Copyright $\odot 2011$ S. Karger AG, Basel 


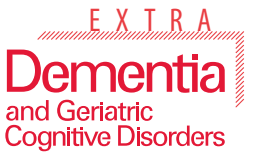

and Geriatric
Cognitive Disorders

\section{Introduction}

Although cognitive function tends to decline with advancing age, the variability ranges from successful aging to dementia. The age-specific prevalence of dementia almost doubles every 5 years, from approximately $1.5 \%$ in persons aged $60-69$ years to $40 \%$ in nonagenarians $[1,2]$. Mild cognitive impairment (MCI; measurable cognitive deficits including deficits in memory, attention, language, visuospatial skill, perceptual speed, or executive functioning, but no dementia) is twice as prevalent as dementia and is associated with an increased risk of dementia $[3,4]$.

Historically, clock-drawing tasks (CDTs) have been used clinically to assess the mental status of neurological and psychiatric patients [5]. Recently, CDTs have been suggested as measures to discriminate between patients with dementia and related disorders and normal subjects [5-7] as well as to assess cognitive function within the normal range [5, 8]. A few studies have shown that the clock-drawing ability of both demented patients $[9,10]$ and healthy elderly $[11,12]$ deteriorates over time.

Few studies of normal elderly participants have looked at CDTs in large or populationbased samples and even fewer have assessed CDTs in the same individuals at different times. Therefore, we analyzed CDTs completed in 2 postal surveys in a large, longitudinal, population-based study of a California retirement community of older men and women.

\section{Methods}

The Leisure World Cohort Study was established in the early 1980s when 13,798 residents of a retirement community (Leisure World Laguna Hills, Calif., USA) completed a postal health survey [13]. The population and the cohort are mostly Caucasian, well-educated, upper-middle class, and elderly; two thirds are women. The initial health survey asked for demographic information, brief medical history, medication use, and lifestyle habits, including cigarette smoking, exercise, and alcohol consumption. The Institutional Review Board of the Keck School of Medicine of the University of Southern California approved this project.

Cohort members were followed by periodic resurveys (1983, 1985, 1992, and 1998), review of local hospital discharge data, and determination of vital status by search of death indexes and ascertainment of death certificates. In 1992, each living cohort member was mailed a follow-up questionnaire. Non-respondents were mailed a second and third questionnaire, and, if a current telephone number was available, were called to encourage participation and/or to complete a telephone interview. In 1998, the cohort members still alive were mailed another follow-up questionnaire. Non-respondents were mailed a second and third questionnaire.

The two follow-up questionnaires (1992 and 1998) included a CDT. A pre-drawn circle with a diameter of $8.3 \mathrm{~cm}$ was provided with the following instructions: 'In the circle below, draw in the numbers as on a clock face.' Following a procedure adapted from Freedman et al. [5] and previously reported by us $[14,15]$, clocks were scored on 7 items: numbers 1-12 present without adding extra numbers or omitting numbers, sequencing of numbers, position of numbers, orientation of numbers to the circle, consistency of Arabic/Roman number representation, tilt of numbers, and superfluous marks [for details on scoring, see ref. 14]. The total clock score is the number of correct items. Clock scoring was done by L.J.C. who was blinded to all other data variables.

$\chi^{2}$ tests were used for comparison of categorical variables and analysis of variance tests for testing differences in means of continuous variables. For multiple comparison of means, 


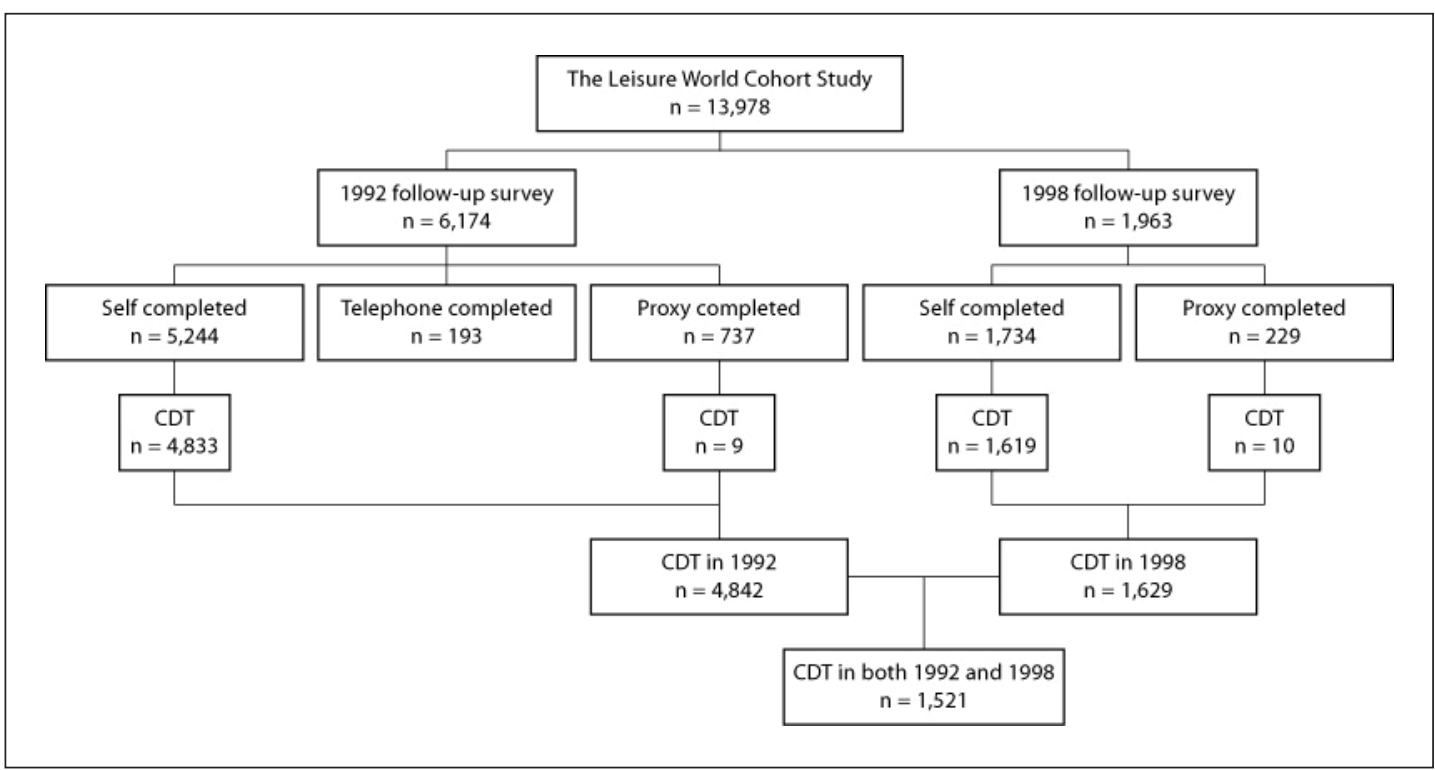

Fig. 1. Leisure World Cohort members who returned the 1992 and 1998 follow-up surveys and who completed the CDT.

Tukey's method for testing pair-wise differences was employed. Relative risks of all-cause mortality and of dementia associated with the CDT were estimated using Cox proportional hazard regression analysis with age at study entry being the age when the 1992 CDT was completed and the event of interest being age at death or dementia. Dementia cases were identified using in-person evaluations which were part of a dementia study [2], hospital records, death certificates, and/or follow-up questionnaires with the date of diagnosis being the date at which dementia was first mentioned.

\section{Results}

Of the 8,403 cohort members who were sent the 1992 survey, 6,174 returned it, 184 were too ill to participate, 388 died within the year without completing the form, 523 refused, and 1,134 did not respond (1,115 known to be alive). Of the 6,174 completed follow-up questionnaires, 4,842 had clocks drawn (fig. 1). Although 9 proxy-completed surveys had a CDT completed by the subject, clocks were not available for the remaining 728 proxy-completed surveys, for the 193 telephone-completed surveys, and for 411 self-completed surveys.

Of the 5,042 cohort members who were sent the 1998 survey, 1,963 returned it, 436 died within the year without completing the form, and 2,643 did not respond (2,560 known to be alive). (Numbers have been updated since our previous report [15] was published.) Of the 1,963 completed surveys, 1,734 were completed by the subjects, including 1,619 with a CDT; 10 of the 229 proxy-completed surveys indicated that subjects had completed the CDT themselves (fig. 1). For these 1,629 completed clocks, a completed clock on the 1992 survey was available for 1,521 subjects. Only 108 subjects (7\%) had no previous CDT: 58 completed the 1992 survey but had no CDT, 6 completed a telephone survey, 12 had a proxy respondent, and 32 did not return the 1992 survey.

Those who completed neither the 1992 nor 1998 CDT were more likely to be older and to have reported on the baseline survey to have chronic disease (high blood pressure, angina, 


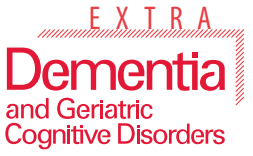

\begin{tabular}{l|l}
\hline \multicolumn{2}{l}{ Dement Geriatr Cogn Disord Extra 2011;1:75-83 } \\
\hline DOI: $10.1159 / 000326781$ & $\begin{array}{l}\text { @ 2011 S. Karger AG, Basel } \\
\text { www.karger.com/dee }\end{array}$ \\
Published online: April 1, 2011 &
\end{tabular}

Paganini-Hill et al.: Longitudinal Assessment of Cognitive Function by CDT

Table 1. CDT scores in the 1992 survey according to 1998 response outcome: the Leisure World Cohort Study

\begin{tabular}{lrl}
\hline 1998 survey response & $\mathrm{n}$ & $\begin{array}{l}1992 \text { clock score } \\
(\text { mean } \pm \text { SD) }\end{array}$ \\
\hline Dead before survey & 1,173 & $5.98 \pm 0.98$ \\
No current address & 65 & $6.17 \pm 0.74$ \\
No survey completed & 1,870 & $6.08 \pm 0.89$ \\
Proxy-completed survey with no CDT & 122 & $5.92 \pm 0.96$ \\
Self-completed survey with no CDT & 91 & $6.05 \pm 0.90$ \\
CDT completed (10 on proxy survey) & 1,521 & $6.30 \pm 0.79$ \\
\hline
\end{tabular}

$\mathrm{p}<0.0001$ for CDT completed vs. all other groups except those with no current address.

heart attack, heart disease, stroke, diabetes, fracture, cataract surgery, and glaucoma), take medication (high blood pressure medication, digitalis, or non-prescription aspirin-containing medication), and exercise less.

Table 1 compares the mean CDT scores in 1992 among the 1998 survey response groups. The mean scores differed significantly among the response groups $(p<0.0001)$ even after adjustment for age and sex. Those who completed both CDTs had the highest mean CDT score in $1992(6.30 \pm 0.79)$, which differed significantly from that of all other groups except those with no current address.

Age, but not sex, had a highly significant effect on CDT scores. Table 2 presents the mean CDT scores in 1992 and 1998, and changes in CDT scores by age group. (Only 2 of the 1,521 subjects who completed both CDTs were identified as demented in 1992.) In both surveys, the mean total scores and the percentage of subjects with correctly drawn clocks decreased with each successive 5 -year age group $(\mathrm{p}=0.0001)$. Pair-wise comparisons between age groups revealed that CDT scores of $\geq 90$-year-old subjects declined significantly more than those of subjects aged $80-84$ years.

Most participants completed the CDT with little or no error on both surveys. About $90 \%$ or more of the subjects produced clocks that were correct in at least 6 of 7 components in both surveys. They placed the numbers 1-12 on their clocks without omissions or additions, had the correct sequencing of numbers, oriented the numbers correctly to the circle (including placing the numbers inside the circle and at a consistent distance from it), used only Arabic or only Roman numerals, had minimal tilt to the numbers, and had no superfluous marks. The most frequent error, made by $>40 \%$ of subjects on each survey, was in positioning the clock numbers. Although a few subjects who had an incorrectly drawn clock in 1992 had a correctly drawn one in 1998 (<3\% of subjects for all components except position of numbers), more subjects with a correctly drawn clock in 1992 made errors in 1998 (4-7\% of subjects for numbers $1-12$, sequence of numbers, and orientation of numbers to the circle).

The 4,828 non-demented subjects who completed the CDT in 1992 were further classified according to their later dementia status. Of the 907 subjects classified as demented, 409 were determined to be demented by clinical evaluation, 31 were identified from hospital records, 435 from death certificates, and 32 from reports by relatives. Of the 1,519 subjects with a second CDT, 22 were newly identified as demented at the time of the second CDT, and an additional 239 subjects were identified as being demented later (1-12 years after the 1998 CDT, median $=8$ years). No dementia was found in the remaining 1,258 subjects. The mean 1992 CDT score of those who became demented by 1998 (6.09 \pm 0.92) was lower, but not 
Table 2. CDT scores according to age: the Leisure World Cohort Study

\begin{tabular}{|c|c|c|c|c|c|}
\hline & \multicolumn{5}{|c|}{ Age in 1998, years } \\
\hline & $<75$ & $75-79$ & $80-84$ & $85-89$ & $\geq 90$ \\
\hline Subjects, n & 156 & 302 & 468 & 389 & 206 \\
\hline \multicolumn{6}{|l|}{1992 CDT score } \\
\hline Mean & 6.47 & 6.40 & 6.30 & 6.26 & 6.08 \\
\hline $\mathrm{SD}$ & 0.69 & 0.76 & 0.74 & 0.80 & 0.93 \\
\hline$\%$ correct & 54 & 51 & 43 & 39 & 34 \\
\hline \multicolumn{6}{|l|}{1998 CDT score } \\
\hline Mean & 6.50 & 6.37 & 6.33 & 6.09 & 5.82 \\
\hline $\mathrm{SD}$ & 0.79 & 0.87 & 0.86 & 1.01 & 1.25 \\
\hline$\%$ correct & 63 & 52 & 50 & 38 & 33 \\
\hline \multicolumn{6}{|l|}{ Change in CDT score } \\
\hline Mean & 0.03 & -0.04 & 0.03 & -0.16 & -0.27 \\
\hline $\mathrm{SD}$ & 0.77 & 1.04 & 1.00 & 1.18 & 1.30 \\
\hline$\%$ with worse score & 19 & 23 & 23 & 31 & 31 \\
\hline \multicolumn{6}{|c|}{$\begin{array}{l}\mathrm{p}<0.05 \text { for participants } \geq 90 \text { vs. }<75,75-79, \text { and } 80-84 \text { years old and } 85-89 \text { vs. }<75 \text { years old for } 1992 \\
\text { mean total CDT scores. } \\
\text { p }<0.01 \text { for participants } \geq 90 \text { years old vs. all other groups and } 85-89 \text { years old vs. all other groups for } \\
1998 \text { mean total CDT scores. } \\
\text { p }<0.05 \text { for participants } \geq 90 \text { vs. } 80-84 \text { years old for change in CDT score. }\end{array}$} \\
\hline
\end{tabular}

Table 3. CDT scores according to dementia status: the Leisure World Cohort Study

\begin{tabular}{lrlll}
\hline & $\mathrm{n}$ & \multicolumn{2}{l}{ CDT scores } & \\
\cline { 3 - 4 } & & \multicolumn{1}{l}{1992} & 1998 & change \\
\hline Dementia in 1998 & 22 & $6.09 \pm 0.92$ & $5.36 \pm 1.53$ & $-0.73 \pm 1.35$ \\
Dementia later & 239 & $6.26 \pm 0.74$ & $6.16 \pm 0.97$ & $-0.10 \pm 1.10$ \\
No dementia known & 1,258 & $6.31 \pm 0.80$ & $6.25 \pm 0.96$ & $-0.06 \pm 1.08$ \\
\hline
\end{tabular}

$\mathrm{p}<0.001$ for difference in 1998 CDT score between the 'dementia in 1998' and both 'dementia later' and 'no dementia known' groups.

$\mathrm{p}<0.03$ for difference in change in CDT score between the 'dementia in 1998' and both 'dementia later' and 'no dementia known' groups.

$\mathrm{p}<0.02$ for change in CDT score different from 0 (no change) in the 'dementia in 1998' group.

significantly, compared with those who became demented later $(6.26 \pm 0.74, \mathrm{p}<0.7)$ or remained non-demented $(6.31 \pm 0.80, \mathrm{p}<0.4)$. The 1998 mean CDT score in the 22 subjects with dementia had declined by 0.73 points $(\mathrm{p}=0.02)$, while the decline in both subjects who became demented later and subjects who remained non-demented was small (table 3).

The age-adjusted risks of both dementia and death increased with decreasing CDT score among the 4,828 subjects who were not demented in 1992 (table 4). Subjects who scored $\leq 5$ in 1992 had a $28 \%$ increased risk of dementia and a 13\% increased risk of death compared with subjects with a perfectly drawn clock. These risks were unchanged when adjusted to other potential risk factors related to mortality in this cohort (smoking, alcohol consumption, activity level, body mass index, and 7 individual histories of high blood pressure, angina, heart attack, stroke, diabetes, rheumatoid arthritis, and cancer). 
Table 4. Relative risks (RRs) of dementia and of death according to CDT scores in 1992: the Leisure World Cohort Study, 1992-2009

\begin{tabular}{lllllrlr}
\hline $\begin{array}{l}\text { Total CDT } \\
\text { score }\end{array}$ & $\begin{array}{l}\text { Subjects } \\
\mathrm{n}\end{array}$ & $\begin{array}{l}\text { Demented } \\
\text { patients, } \mathrm{n}\end{array}$ & RR & $95 \% \mathrm{CI}$ & Dead, $\mathrm{R}$ & $\mathrm{RR}$ & 95\% CI \\
\hline 7 (perfect) & 1,692 & 272 & 1.00 & & 1,319 & 1.00 & \\
6 & 2,413 & 487 & 1.20 & $1.03-1.39$ & 2,033 & 1.06 & $0.99-1.14$ \\
$\leq 5$ & 723 & 148 & 1.28 & $1.05-1.57$ & 640 & 1.13 & $1.03-1.25$ \\
\hline
\end{tabular}

\section{Discussion}

Accurate clock drawing involves a diverse range of higher-level cognitive abilities [5, reviewed in ref. 6 and 16]. It requires long-term and working memory, visuospatial abilities, motor execution, language comprehension, numerical knowledge, attention, abstract thinking, and executive functions. The drawer must possess language skills to comprehend the instructions, have a representation of the visuospatial features of a clock, be able to retrieve this information from memory, and have visual perception to maintain the correct spatial layout of the clock. The wide range of cognitive functions and especially the executive control measures tapped by a CDT may make it more sensitive to cognitive impairment than traditional screening measures in the well elderly [17].

Our study showed that although most elderly adults can complete a CDT with little or no error, the proportion of subjects with errors increased over time, and those who later were identified as demented had lower CDT scores than those who remained non-demented. Similarly, in a study of 427 Italian men (mean age 78 years, range 72-90 years) followed for 4 years, a CDT identified older persons at a high risk of cognitive decline [8]. A rural Pennsylvanian community-based prospective study - Monongahela Valley Independent Elders Survey (MoVIES Project) - examined 1,017 non-demented subjects (mean age 74 years) at baseline and 2 years later [11]. The mean CDT score declined only slightly, suggesting overall stability of performance over 2 years; however, standard deviations were large, implying considerable individual variation. Continued 10-year follow-up in this study allowed comparison of 68 individuals later identified as having clinical Alzheimer's disease (AD) with 483 non-demented controls on cognitive tests completed 2 years apart, and for cases 3.5 and 1.5 years before diagnosis [12]. Mean decline in the CDT was greater in cases than controls, but the difference was not statistically significant $(\mathrm{p}=0.11)$. Also in the MoVIES study, a factor analysis of 42510 -year survivors identified constructional praxis (including the CDT) as impacting cognitive decline [18]. In another study of 59 subjects seen at an AD clinic but not meeting diagnostic criteria for dementia at their initial assessment, the 22 subjects diagnosed as demented on their second visit had lower CDT scores (primarily due to number substitutions) at baseline [19]. Likewise, at a memory clinic, 123 patients were grouped by eventual cognitive status: AD patients $(\mathrm{n}=16)$, MCI patients progressing to dementia (mean duration 4.1 years; $\mathrm{n}=22)$, MCI patients not progressing to dementia $(\mathrm{n}=49)$, and normal subjects $(\mathrm{n}=76)$ [20]. The mean CDT scores decreased with increasing cognitive problems. The AD group scored significantly worse than the other groups; the MCI progressors scored significantly worse than the normal group, but the MCI progressors and the MCI non-progressors did not differ. However, MCI progressors and MCI non-progressors differed in the drawing and placement of the hands. In another study of 25 healthy elderly adults living in a senior residence, the majority of poorly drawn clocks were produced by subjects with lower (although normal) scores on the Dementia Rating Scale [5]. Comparison of these adults with 
their community-dwelling counterparts found poorer positioning of numbers in the residential sample, suggesting poor clock drawing may be an early sign of cognitive decline.

Although CDT scores decline with age and the CDT helps to discriminate between demented and non-demented subjects, the greatest usefulness of the CDT would be to identify subjects with MCI - the main target for early interventions to delay dementia. The suitability of the CDT for this remains unclear [21]. Although we did not focus on MCI, our results showing lower CDT scores in non-demented individuals who later became demented add some support to the notion that a CDT may help identify individuals at an increased risk of dementia.

We also observed that a worse CDT score was associated with an increased risk of allcause mortality. Inclusion of health indicators, such as history of chronic diseases, smoking, and alcohol intake, did not markedly change the observed relative risk. Cognitive impairment as measured by the CDT seemed to be an independent risk factor for mortality in this population. Three other studies have found this relationship with mortality [22-24]. Data from the MRC Trial of the Assessment and Management of Older People in the Community (aged $\geq 75$ years) revealed a $75 \%$ increased risk of death among subjects with poor CDT scores [22]. Risk increased with decreasing scores among the 13,557 subjects followed for a median of 4.6 years. In 230 residents (aged 69-99 years) of a continuing-care retirement community in Pennsylvania, low scorers on a CDT had more than twice the risk of death during a 4-year follow-up compared with high scorers [23]. Five-year survival among 242 non-demented residents ( $>70$ years old) of another continuing-care retirement community was related significantly to performance on a CDT [24]; low scores were associated with about 25\% increased risk of death.

Poor performance on constructional tasks including CDTs has previously been found to be related to right temporoparietal changes on neuroimaging of $\mathrm{AD}$ patients [25]. Royall et al. [24] hypothesize that in normal aging, preclinical right-hemisphere AD pathology may be responsible for increased mortality from changes in cortical control of autonomic functions, which may, for example, increase arrhythmias and falls.

Several limitations of our study should be noted. A major limitation of our study is that we did not receive a CDT from the majority of the subjects sent the 1998 follow-up questionnaire. Non-respondents may have chosen not to complete the follow-up surveys due to the nature of the questions and/or their own general health. Selective attrition of the cognitively impaired is a recognized problem in epidemiologic studies [26] and militates against demonstrating associations with cognitive impairment. In a community-based cross-sectional study of cognitive function in the Netherlands, non-respondents were more likely to perform poorly in the cognitive test, especially if they were $<75$ years old [27]. In addition, as our tests were mailed to the patients and completed at home, we cannot be sure whether the tests were completed by the participants on their own as instructed or with guidance from someone else. However, clocks drawn at home correlate with clocks drawn in the presence of an examiner $(r=0.83)$ [Stanley Birge, pers. commun.]. Additionally, the proportion of demented individuals is likely an underestimation of the true prevalence, and we lacked clinical diagnoses of dementia based on standard criteria for many subjects. For these reasons, our interpretation of this cognitive assessment is preliminary and incomplete. Furthermore, our subjects are from a very select population - moderately affluent, highly educated, health conscious, and primarily Caucasian. Thus, the generalizability of our results is limited and may not apply to more ethnically diverse, lower-income and less well-educated populations.

Our CDT did not include asking the subjects to draw the hands of the clock at a specific time setting (such as $10 \mathrm{~min}$ past 11). Drawing the hands may provide additional information on cognition and is included in some scoring methods [see ref. 7 for a list of CDT scoring systems]. Tuokko et al. [28] observed that the omission of hands was more frequent in AD pa- 
tients than normal elderly subjects. Similarly, Esteban-Santillan et al. [29] found that the placement of hands on the clock provides very high positive and negative predictive values for early AD. Others, however, have found that hand placement correlates with other components of the CDT and does not add substantially to its discriminating power [9, 30, 31].

The rapidly growing numbers and proportions of the very old, in whom the prevalence of cognitive impairment is highest, highlight the need for efficient and effective cognitive screening methods [32]. Clock drawing is an appealing measure of cognitive function because it is quick and easy to administer and score, is acceptable by subjects, does not require in-person assessment, has good inter-rater and test-retest reliability, correlates with more detailed and time-consuming cognitive screens, and is relatively free of influence by language, cultural, or ethnic factors [6]. The associations of low and declining CDT scores with the later development of dementia suggest that small changes in the CDT over time may be a sign of early cognitive change. Further investigation of patients with such changes may lead to an early diagnosis of MCI and consideration of therapy and future life-planning of impaired individuals.

\section{Acknowledgment}

This research was funded by grants from the National Institutes of Health (R01CA32197 and P50AG05142), the Errol Carroll Trust Fund, and Wyeth-Ayerst Laboratories.

\section{References}

$\checkmark 1$ Qiu C, De Ronchi D, Fratiglioni L: The epidemiology of the dementias: an update. Curr Opin Psychiatry 2007;20:380-385.

-2 Corrada MM, Brookmeyer R, Berlau D, Paganini-Hill A, Kawas CH: Prevalence of dementia after age 90: results from the 90+ study. Neurology 2008;71:337-343.

-3 Winblad B, Palmer K, Kivipelto M, et al: Mild cognitive impairment - beyond controversies, towards a consensus: report of the International Working Group on Mild Cognitive Impairment. J Intern Med 2004;256:240-246.

4 Bischkopf J, Busse A, Angermeyer MC: Mild cognitive impairment - a review of prevalence, incidence and outcome according to current approaches. Acta Psychiatr Scand 2002;106:403-414.

5 Freedman M, Leach L, Kaplan E, Winocur G, Shulman KI, Delis DC: Clock Drawing. A Neuropsychological Analysis. New York, Oxford University Press, 1994.

-6 Shulman KI: Clock-drawing: is it the ideal cognitive screening test? Int J Geriatr Psychiatry 2000;15: 548-561.

7 Pinto E, Peters R: Literature review of the Clock Drawing Test as a tool for cognitive screening. Dement Geriatr Cogn Disord 2009;27:201-213.

8 Ferrucci L, Cecchi F, Guralnik JM, Giampaoli S, Noce CL, Salani B, Bandinelli S, Baroni A, for the FINE Study Group: Does the clock drawing test predict cognitive decline in older persons independent of the Mini-Mental State Examination? J Am Geriatr Soc 1996;44:1326-1331.

-9 Rouleau I, Salmon DP, Butters N, Kennedy C, McGuire K: Quantitative and qualitative analyses of clock drawings in Alzheimer's and Huntington's disease. Brain Cogn 1992;18:70-87.

-10 Shulman KI, Gold DP, Cohen CA, Zucchero CA: Clock-drawing and dementia in the community: a longitudinal study. Int J Geriatr Psychiatry 1993;8:487-496.

-11 Ganguli M, Seaberg EC, Ratcliff GC, Belle SH, DeKosky ST: Cognitive stability over 2 years in a rural elderly population: the MoVIES Project. Neuroepidemiology 1996;15:42-50.

12 Chen P, Ratcliff G, Belle SH, Cauley JA, DeKosky ST, Ganguli M: Patterns of cognitive decline in presymptomatic Alzheimer disease. A prospective community study. Arch Gen Psychiatry 2001;58: 853-858. 
13 Paganini-Hill A, Chao A, Ross RK, Henderson BE: Exercise and other factors in the prevention of hip fracture: the Leisure World Study. Epidemiology 1991;2:16-25.

-14 Paganini-Hill A, Clark LJ, Henderson VW, Birge SJ: Clock drawing: analysis in a retirement community. J Am Geriatr Soc 2001;49:941-947.

-15 Paganini-Hill A, Clark LJ: Preliminary assessment of cognitive function in older adults by clock drawing, box copying and narrative writing. Dement Geriatr Cogn Disord 2007;23:74-81.

16 Peters R, Pinto EM: Predictive value of the clock drawing test. A review of the literature. Dement Geriatr Cogn Disord 2008;26:351-355.

-17 Román GC, Royall DR: Executive control function: a rational basis for the diagnosis of vascular dementia. Alzheimer Dis Assoc Disord 1999;13(suppl 3):S69-S80.

- 18 Ratcliff G, Dodge H, Birzescu M, Ganguli M: Tracking cognitive functioning over time: ten-year longitudinal data from a community-based study. Appl Neuropsychol 2003;10:76-88.

19 O'Rourke N, Tuokko H, Hayden S, Beattie BL: Early identification of dementia: predictive validity of the clock test. Arch Clin Neuropsychol 1997;12:257-267.

-20 Babins L, Slater ME, Whitehead V, Chertkow H: Can an 18-point clock-drawing scoring system predict dementia in elderly individuals with mild cognitive impairment? J Clin Exp Neuropsychol 2008; 30:173-186.

-21 Ehreke L, Luppa M, König HH, Riedel-Heller SG: Is the clock drawing test a screening tool for the diagnosis of mild cognitive impairment? A systematic review. Int Psychogeriatr 2010;22:56-63.

-22 Nishiwaki Y, Breeze E, Smeeth L, Bulpitt CH, Peters R, Fletcher AE: Validity of the clock-drawing test as a screening tool for cognitive impairment in the elderly. Am J Epidemiol 2004;160:797-807.

-23 Lavery LL, Starenchak SM, Flynn WB, Stoeff MA, Schaffner R, Newman AB: The Clock Drawing Test is an independent predictor of incident use of 24-hour care in a retirement community. J Gerontol A Biol Sci Med Sci 2005;60:928-932.

-24 Royall DR, Chioda LK, Mouton C, Polk MJ: Cognitive predictors of mortality in elderly retirees: results from the Freedom House Study. Am J Geriatr Psychiatry 2007;15:243-251.

-25 Cahn-Weiner DA, Sullivan EV, Shear PK, Fama R, Lim KO, Yesavage JA, Tinklenberg JR, Pfefferbaum A: Brain structural and cognitive correlates of clock drawing performance in Alzheimer's disease. J Int Neuropsychol Soc 1999;5:502-509.

26 Bari MD, Williamson J, Pahor M: Missing data in epidemiological studies of age-associated cognitive decline. J Am Geriatr Soc 1999;47:1380-1381.

27 Launer LJ, Wind AW, Deeg DJH: Nonresponse pattern and bias in a community-based cross-sectional study of cognitive functioning among the elderly. Am J Epidemiol 1994;139:803-812.

-28 Tuokko H, Hadjistavropoulos T, Miller JA, Beattie BL: The clock test: a sensitive measure to differentiate normal elderly from those with Alzheimer's disease. J Am Geriatr Soc 1992;40:579-584.

-29 Esteban-Santillan C, Praditsuwan R, Ueda H, Geldmacher DS: Clock drawing test in very mild Alzheimer's disease. J Am Geriatr Soc 1998;46:1266-1269.

-30 Mendez MF, Ala T, Underwood KL: Development of scoring criteria for the clock drawing task in Alzheimer's disease. J Am Geriatr Soc 1992;40:1095-1099.

31 Tsering C, Miller JP, Morris JC, et al: Relationships of features of clocks drawn to other cognitive measures in the OASIS study cohort. 47th Annual Meeting of the American Geriatric Society, Atlanta, 1990.

-32 Malloy PF, Cummings JL, Coffey CE, et al: Cognitive screening instruments in neuropsychiatry: a report of the Committee on Research of the American Neuropsychiatric Association. J Neuropsychiatry Clin Neurosci 1997;9:189-197. 